\title{
Treatment of aerodigestive fistulas with a novel covered metallic Y-shaped segmented airway stent customized with the assistance of 3D printing
}

\author{
Qungang Shan ${ }^{1 \#}$, Wei Huang ${ }^{1 \#}$, Mingyi Shang ${ }^{2}$, Ziyin Wang ${ }^{1}, \mathrm{Ning}_{\mathrm{Xia}}{ }^{3}$, Qingsheng Xue ${ }^{4}$, Aiwu Mao ${ }^{2}$, \\ Xiaoyi Ding ${ }^{1}$, Zhongmin Wang ${ }^{1,3}$
}

${ }^{1}$ Department of Interventional Radiology, Ruijin Hospital, Shanghai Jiao Tong University School of Medicine, Shanghai, China; ${ }^{2}$ Department of Interventional Radiology, Tongren Hospital, Shanghai Jiao Tong University School of Medicine, Shanghai, China; ${ }^{3}$ Department of Radiology, Ruijin Hospital/Luwan Branch, Shanghai Jiao Tong University School of Medicine, Shanghai, China; ${ }^{4}$ Department of Anesthesiology, Ruijin Hospital, Shanghai Jiao Tong University School of Medicine, Shanghai, China

Contributions: (I) Conception and design: Z Wang, Q Shan, W Huang; (II) Administrative support: Z Wang, Q Shan, W Huang, M Shang; (III) Provision of study materials or patients: Z Wang, Q Shan, W Huang, M Shang; (IV) Collection and assembly of data: All authors; (V) Data analysis and interpretation: All authors; (VI) Manuscript writing: All authors; (VII) Final approval of manuscript: All authors.

\#These authors contributed equally to this work.

Correspondence to: Zhongmin Wang. Department of Interventional Radiology, Ruijin Hospital, Shanghai Jiao Tong University School of Medicine, No. 197, Ruijin 2nd Road, Huangpu District, Shanghai 200025, China. Email: wzm11896@rjh.com.cn.

Background: The management of aerodigestive fistula remains challenging. An airway stent that matches well with the individual geometry of the airway is needed for the treatment of the aerodigestive fistula. This study aimed to evaluate the feasibility of a novel covered metallic segmented Y-shaped airway stent customized with the assistance of $3 \mathrm{D}$ printing in aerodigestive fistulas involving the carina and distal bronchi and to compare the flexibility of the novel stent with the conventional wholly knitted stent.

Methods: In the flexibility study, we measured the longitudinal bending force and spring-back force of the segmented stent and wholly knitted stent. Patient-specific stents that were individually customized with the assistance of 3D printing technology were implanted in 26 patients with aerodigestive fistulas. The technical success, clinical success, Karnofsky performance status (KPS), and stent-related complications were recorded. Results: The bending force and spring-back force of the segmented stent were significantly lower than those of the wholly knitted stent. Stent deployment was technically successful in all patients. Clinical success was obtained in 21 patients. The KPS of patients after the stenting procedure improved significantly compared with that before stenting $(\mathrm{P}<0.001)$. During follow-up, granulation tissue proliferation, sputum retention, stent migration, and intolerance of the stent were found in 2, 5, 1, and 1 patient, respectively.

Conclusions: The segmented metallic Y-shaped airway stent had greater flexibility than the wholly knitted stent in an ex vivo setting. Implantation of the segmented stent individually customized with the aid of 3D printing is feasible in treating aerodigestive fistulas involving the carina and bronchi distal to the carina.

Keywords: Carina; airway fistula; stent; 3D printing

Submitted Feb 14, 2021. Accepted for publication May 02, 2021.

doi: $10.21037 / \mathrm{atm}-21-733$

View this article at: https://dx.doi.org/10.21037/atm-21-733 


\section{Introduction}

Aerodigestive fistula is a severe complication related to malignant tumors such as lung cancer and esophageal carcinoma, or other etiologies, including surgery, radiation, and trauma $(1,2)$. The incidence of aerodigestive fistulas in patients with esophageal carcinoma and lung cancer was reported to be $5-15 \%$ and $1 \%$, respectively $(1,3)$. The mortality rate of the aerodigestive fistula is high due to lethal infection, and its treatment remains challenging $(4,5)$. Patients with aerodigestive fistulas often have a refractory infection and may be too debilitated to tolerate surgery, and the outcome of conservative treatments is poor $(1,6)$. Therefore, a minimally invasive and effective treatment modality for aerodigestive fistulas is needed.

Various kinds of airway stents have been widely used in treating aerodigestive fistulas in the clinic because of their safety, efficacy, and minimal invasiveness (7-10). Y-shaped airway stents have been currently used for fistulas involving the carina and distal bronchi $(7,8,11)$. The geometry of the carina and distal bronchi is complicated and variable among different patients. The current commercial Y-shaped stent may not fit the airway of patients, thus increasing stent-related complications. Therefore, patient-specific airway stents manufactured according to the anatomical features of the airway are needed $(7,12,13)$. Advances in $3 \mathrm{D}$ printing offer support for designing patient-specific airway stents. A patient-specific airway stent can be created using a $3 \mathrm{D}$ printing mold made according to the anatomical information of the airway captured by computed tomography (CT) to fit the individual geometry of the airway (9,14-17).

The flexibility of the stent is defined as the ease of bending and deformation of the stent, and it determines the ability of the stent to adapt to changes in the shape of the lumen (18-21). The flexibility of stents can be evaluated by the measurement of bending force and springback force (20,22-24). Previous studies have reported that adverse events after stent implantation were related to poor flexibility of the stent in the fields of biliary stents and coronary stents $(20,24)$. The same reasoning applies to the Y-shaped airway stent. For the Y-shaped airway stent, smaller bending force and spring-back force mean higher stent flexibility and a stronger ability to adapt to dynamic changes of the bronchus. After the Y-shaped airway stent is implanted, the angle of the stent may be inconsistent with the tracheal carina angle during deep inhalation and exhalation or when a tumor compresses the airway, and the spring-back force of the stent will act on the wall of the bronchus $(25,26)$. The mechanical stimulation to the airway wall after airway stenting is an important factor leading to complications such as granulation hyperplasia, airway wall injury, and patient intolerance $(27,28)$. Therefore, the stent should have good flexibility to reduce the mechanical stimulation on the airway wall. To increase the flexibility of the airway stent, we designed a novel stent that is segmented at the connection of the stent. However, the quantitative characterization of the flexibility of the Y-shaped airway stent is complex and currently lacking, and the optimal flexibility of the stent remains unclear.

Furthermore, a comparison of the flexibility of the novel stent and the conventional wholly knitted stent has not been reported. This study aimed to evaluate the feasibility of the treatment of aerodigestive fistulas involving the carina and distal bronchi using the novel covered metallic segmented Y-shaped airway stent customized with the assistance of $3 \mathrm{D}$ printing and to compare the flexibility of the segmented stent with the wholly knitted stent. We present the following article in accordance with the STROBE (Strengthening the Reporting of Observational Studies in Epidemiology) reporting checklist (available at https:// dx.doi.org/10.21037/atm-21-733).

\section{Methods}

\section{Patients}

The study was conducted following the Declaration of Helsinki (as revised in 2013). The study was approved by the Ethical Committee of the Ruijin Hospital/Luwan Branch (No. LWEC2019018), and individual consent for this retrospective analysis was waived. We retrospectively reviewed data of a consecutive group of patients who underwent airway stenting due to aerodigestive fistulas from January 2017 to March 2020. The inclusion criteria were as follows: patients with aerodigestive fistulas involving the carina or distal bronchi; and patients who underwent insertion of the covered metallic segmented Y-shaped airway stent customized with the assistance of $3 \mathrm{D}$ printing. The exclusion criteria were as follows: patients with airway stenosis; and patients who underwent esophageal stenting. Our institutional review board approved the stent for use in patients with airway fistulas, which were difficult to treat with commercial stents. All patients provided written informed consent before the stenting procedure. The diagnosis of aerodigestive fistulas was confirmed according 
to clinical manifestations, chest CT examination, and bronchoscopy. Gastroscopy was performed if necessary.

\section{Customization of the airway stent}

The chest CT scan data of patients were imported into the $3 \mathrm{D}$ reconstruction system (Vitaworks, Shanghai, China) to reconstruct $3 \mathrm{D}$ images of the airway, which were immediately transferred to a 3D printer (RS600, Union Tech, Shanghai, China). Next, a 1:1 scale airway mold was printed according to each patient's anatomical features using photosensitive resins. Under the 3D airway mold guidance, airway stents (Micro-Tech, Nanjing, China) were woven using the mold as a template. The Y-shaped segmented stent was composed of the main stem, two branches, and a connecting part. The main stem and two branches were woven using nickel titanium alloy wire into a tubular configuration. The connecting part of the stent was woven using polytetrafluoroethylene (PTFE) thread in a meshed manner. The connecting part linked the end of the main stem and branches to form a Y-shaped stent. The stent was fully covered with silicone. The stent dimensions, including diameter, length, and angulation, were properly matched to the airway anatomy of specific patients with the aid of the mold (Figure 1). With the assistance of the airway mold, the fenestration of the stent can match the location and size of the opening of the bronchus, which can guarantee the sealing of the fistula around the opening of the bronchus without occluding the opening. The proximal and distal ends of the stent were labeled with markers to achieve precise placement. The time of stent manufacture was 34 days.

\section{Stenting procedure}

Before stenting, the location and size of the aerodigestive fistulas were confirmed by CT and bronchoscopy. General anesthesia was performed, and mechanical ventilation was maintained through a tracheal catheter (7.0F, Tuoren, China). Under flexible bronchoscopic and fluoroscopic guidance, the stent delivery system was introduced into the airway via 0.035 -inch stiff guide wires (Terumo, Tokyo, Japan). Afterward, the Y-shaped stent was inserted and positioned to seal the fistula, with its distal end at least $1 \mathrm{~cm}$ beyond that of the fistula (Figure 2). CT and flexible bronchoscopy were performed immediately after stenting to confirm the sealing of the fistula and the expansion and position of the stent.

\section{Follow-up}

All patients underwent bronchoscopy, chest CT examination, and contrast esophagography 3-7 days and 1 month after stent implantation. Further followup was performed every 3 months if patients did not show symptoms of aerodigestive fistulas or stent-related complications. Karnofsky performance status (KPS) was used to evaluate the patient's physical condition before and 1 month after stenting. The stent was removed under bronchoscopy with local anesthesia when stent-related complications occurred or when the fistula was healed. Stent removal was performed under local anesthesia. Under the guidance of bronchoscopy, the tip of the removal hook was inserted to hook the retrieval thread on the stent's upper end.

The definition of technical success was the accurate placement of the stent at the targeted region confirmed by CT and bronchoscopy, with no significant complications occurring. Clinical success was defined as complete sealing of the fistula with an improvement of aspiration symptoms. Curing of the fistula was defined as complete healing of the fistula after stent removal. The definition of stent-related complications was complications correlated with the stent after stent placement $(11,29-31)$. During follow-up, the clinical data of all patients were analyzed.

\section{In vitro test of the flexibility of stents}

The segmented and wholly knitted Y-shaped airway stents (the angle of the two branches, $90^{\circ}$; diameter and length of the main stem, 20 and $60 \mathrm{~mm}$, respectively; diameter and length of the branches, 14 and $30 \mathrm{~mm}$, respectively; Micro-Tech, Nanjing, China; $\mathrm{n}=5$ ) were used for the in vitro experiments. All stents were made of nickel titanium alloy wire and fully covered with silicone. All characteristics of the two kinds of stents were the same except for the connection of the main stem and branches of the stents. We measured the longitudinal bending force and springback force of the segmented stent and conventional wholly knitted stent using compression equipment of the biological conduit (LLY-06D, Yantai, China). An oval pressing head with a long diameter of $3 \mathrm{~cm}$ and a short diameter of $2 \mathrm{~cm}$ was connected with the equipment. Each test sample was fixed on a rod that fit the inner diameter of the main stem of the stent. The remaining parts of the stent were kept flexible without fixation. Subsequently, the sample was compressed to a diameter of $20 \mathrm{~mm}$, and the bending 


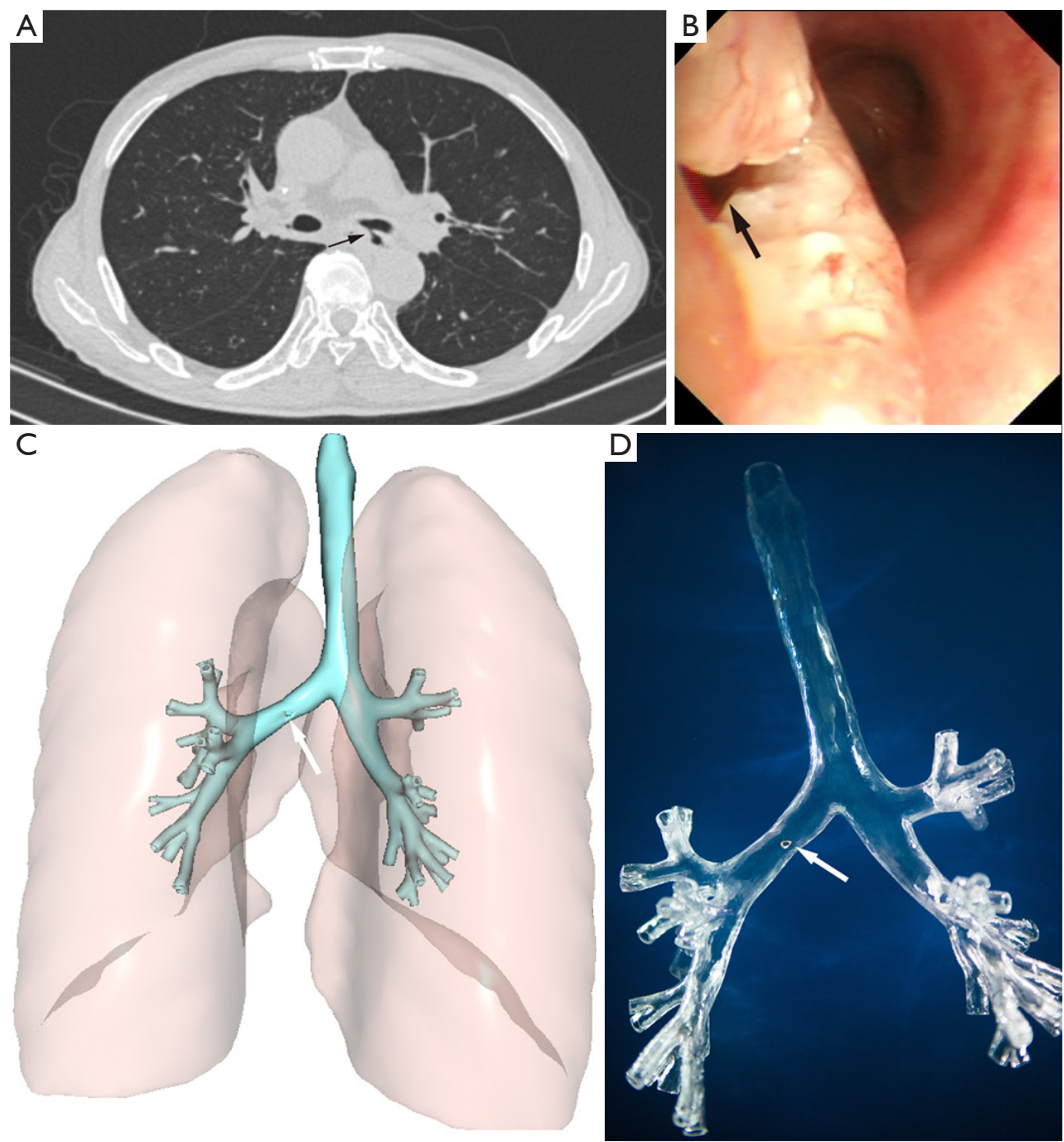

Figure 1 A 65 -year-old man with malignant esophago-left main bronchus fistula caused by invasion of esophageal cancer before stenting. (A) Axial CT image showed that the left main bronchus communicated with the esophagus (black arrow); (B) bronchoscopy showed the fistula located at the posterior wall of the left main bronchus (black arrow); (C) 3D CT reconstruction showed the patient's airway with the fistula (white arrow); (D) the 3D patient-specific mold of the airway was printed by a 3D printer based on CT reconstruction. The mold can clearly show the location and size of the fistula (white arrow). 3D, three-dimensional; CT, computed tomography.

force and spring-back force of each sample were recorded continuously while compressing (Figure 3). The forces of the 2 kinds of stents were compared when the compressing distances were 5, 10, and $15 \mathrm{~mm}$, respectively.

\section{Statistical analysis}

Data for continuous measures were expressed as mean $\pm \mathrm{SD}$ or median [interquartile range (IQR)]. The Wilcoxon test for paired samples was used to compare KPS before and after stent implantation. The other continuous variables were compared using the Wilcoxon test. The Kaplan-Meier method was used to calculate the overall survival. $\mathrm{P}<0.05$ was considered statistically significant. Statistical analyses were performed using SPSS 22.0 (IBM Corp., USA) and GraphPad Prism 6 (GraphPad Software Inc., USA). 

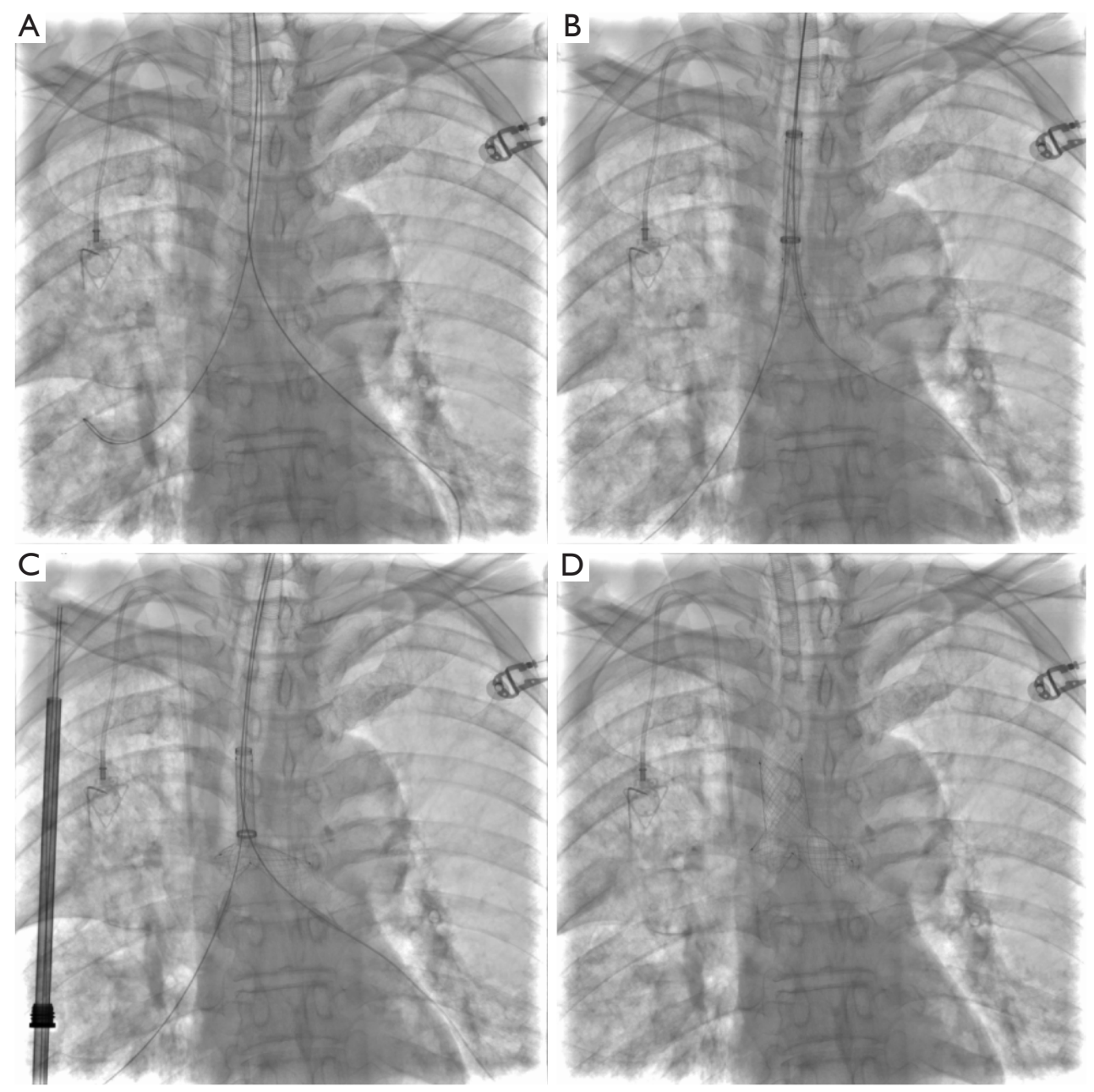

Figure 2 The process of the stenting procedure. (A) Under the guidance of fluoroscopy and flexible bronchoscopy, 2 catheters and soft guidewires were placed into the left and right bronchus. Afterwards, the soft guidewires were replaced by stiff guidewires; (B) under fluoroscopy, the stent sheathed in the delivery system was introduced using the stiff guidewires; (C,D) the stent was released after it was delivered to the targeted position.

\section{Results}

\section{Patient characteristics}

A total of 26 patients ( 24 males and 2 females, mean age $59.42 \pm 8.65$ years, range $38-77$ years) were enrolled for analysis. Ten of the patients had aerodigestive fistulas caused by malignant tumors, such as esophageal carcinoma $(\mathrm{n}=6)$ and lung cancer $(\mathrm{n}=4)$. Sixteen patients were diagnosed with aerodigestive fistulas after esophagectomy. All patients showed aspiration pneumonia and symptoms of irritating cough when eating or drinking. All patients underwent conservative treatments, such as anti-infection treatment and mediastinal drainage before the stenting procedure.
The baseline characteristics of patients are shown in Table 1 .

\section{Outcome and follow-up}

The stenting procedures were technically successful in all patients without procedural complications at a median of 30 (IQR, 19.25-104.25) days after diagnosis of aerodigestive fistulas. The stents demonstrated sufficient expansion according to immediate chest CT and bronchoscopy after stenting. A small Y-shaped segmented stent was implanted in a patient with a fistula located at the left main bronchus (Figure 4). Twenty-one patients had no further aspiration symptoms after the stenting procedure, with a clinical 

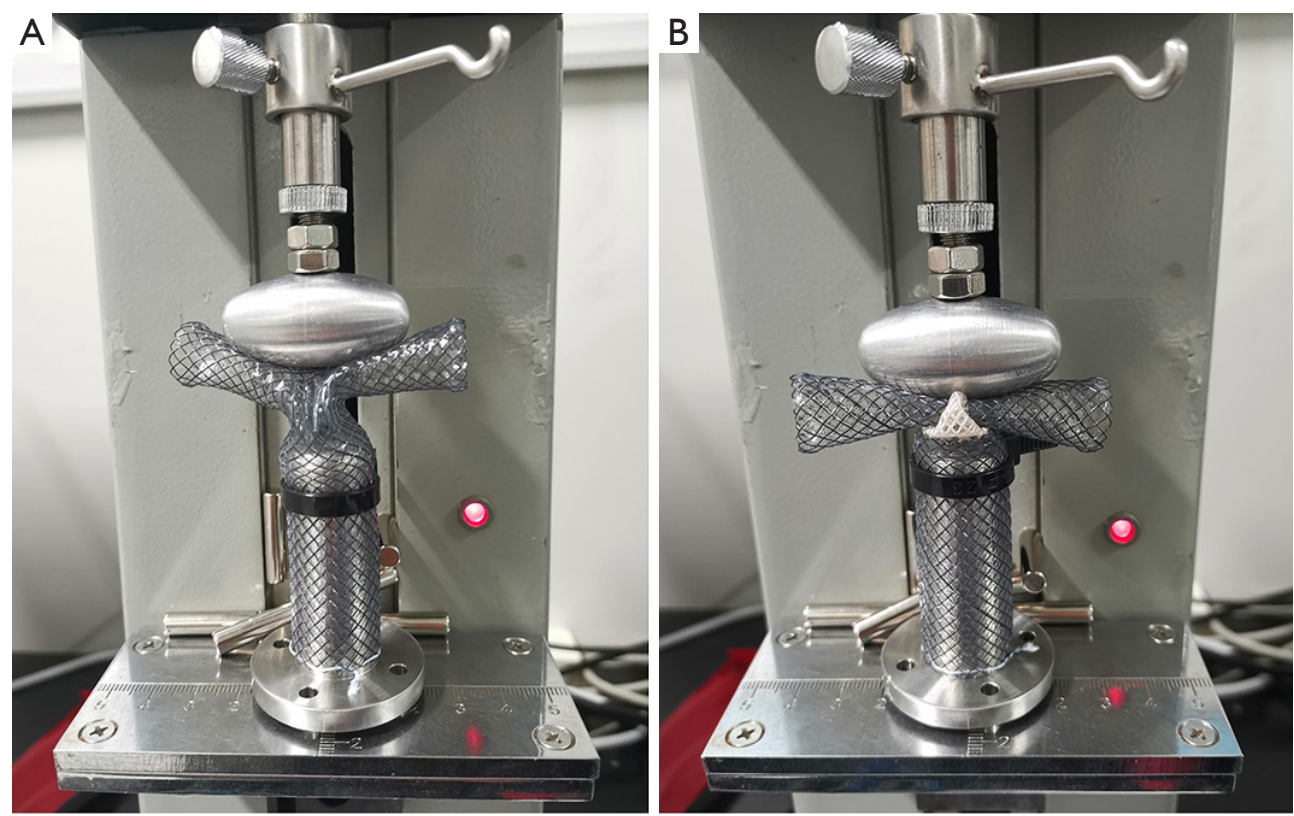

Figure 3 In vitro test of the flexibility of stents. The longitudinal bending force and spring-back force of the conventional wholly knitted stent (A) and segmented stent (B) were measured using compression equipment of the biological conduit.

success rate of $80.77 \%$. Esophagography showed no contrast leaking into the airways in these patients. Esophagography showed contrast agents flowing into the airway via the fistula in the other 5 patients. Aerodigestive fistulas were cured in 9 of the 16 patients after esophagectomy, and the stents were removed (Figure 5). None of the malignant fistulas were cured. In total, $34.6 \%$ of all patients achieved healing. For benign aerodigestive fistulas with and without healing, the time interval between diagnosis of the fistula and the airway stenting procedure was 30 (IQR, 18.5-55.5) and 117 (IQR, 35-240) days, respectively $(\mathrm{P}=0.017)$. The KPS of patients after the stenting procedure improved significantly compared with that before stenting [70 (IQR, 70-80) vs. 60 (IQR, 57.5-60); $\mathrm{P}<0.001]$.

The median follow-up time of patients was 150 (IQR, 100-267.5) days. During follow-up, 2 (7.69\%) patients had granulation tissue proliferation, which was treated successfully by cryotherapy via bronchoscopy and stent removal. Five $(19.23 \%)$ patients had sputum retention, which was treated by sputum aspiration under bronchoscopy. One $(3.84 \%)$ patient had stent migration and showed manifestations of aerodigestive fistulas during follow-up, and the patient underwent a second stenting procedure after retrieval of the former stents. A stent was removed in $1(3.84 \%)$ patient who could not tolerate the stent. After the severe clinical symptoms improved and general health was recovered, patients underwent chemotherapy or radiotherapy. Reopening of the fistula was found in 4 patients with initial complete sealing during follow-up. The median overall survival time was 260 days. The death of patients was due to tumor progression $(n=12)$ and severe infection $(n=4)$, and no patients died due to the stent.

\section{Flexibility of stents}

The bending force of the segmented stent was significantly lower than that of the wholly knitted stent when the compressing distances were 5,10 , and $15 \mathrm{~mm}(82.28 \pm 13.95$ vs. $100.45 \pm 8.74 \mathrm{cN}, \mathrm{P}=0.039 ; 270.83 \pm 16.80$ vs. $432.51 \pm 7.54$ $\mathrm{cN}, \mathrm{P}<0.001 ; 517.11 \pm 42.72$ vs. $750.13 \pm 12.12 \mathrm{cN}, \mathrm{P}<0.001)$. The spring-back force was significantly lower in the segmented stent group than the wholly knitted stent group when the compressing distances were 5,10 , and $15 \mathrm{~mm}$ $(12.44 \pm 2.85$ vs. $51.99 \pm 6.65 \mathrm{cN}, \mathrm{P}<0.001 ; 94.94 \pm 31.41$ vs. $280.69 \pm 16.43 \mathrm{cN}, \mathrm{P}<0.001 ; 333.38 \pm 78.60$ vs. $539.63 \pm$ $13.43 \mathrm{cN}, \mathrm{P}=0.004$ ) (Figure 6). The flexibility of the segmented metallic Y-shaped airway stent was better than that of the wholly knitted stent.

\section{Discussion}

Aerodigestive fistulas are associated with various factors, 
Table 1 Baseline characteristics of patients

\begin{tabular}{lc}
\hline Characteristics & All patients $(\mathrm{n}=26)$ \\
\hline Age (years) & $59.42 \pm 8.65$ \\
Gender, $\mathrm{n}(\%)$ & $24(92.3)$ \\
Male & $2(7.7)$ \\
Female & \\
Previous disease, $\mathrm{n}(\%)$ & $22(84.6)$ \\
Esophageal cancer & $4(15.4)$ \\
Lung cancer & \\
Cause of fistula, $\mathrm{n}(\%)$ & $16(61.5)$ \\
Esophagectomy & $10(38.5)$ \\
Malignancy & \\
Type of fistula, $\mathrm{n}$ (\%) & \\
Esophagotracheal fistulas & $4(15.3)$ \\
Esophagobronchial fistulas & $6(23.1)$ \\
Thoracogastric-tracheal fistulas & $10(38.5)$ \\
Thoracogastric-bronchial fistulas & $6(23.1)$ \\
\hline
\end{tabular}

${ }^{*}$, reported as mean $\pm \mathrm{SD}$.

such as invasion of advanced tumors, or benign etiologies, such as esophagectomy, radiation damage, and iatrogenic injury $(1,5,32)$. Patients with aerodigestive fistulas may be too debilitated to tolerate surgical repair, and the outcome of conservative treatment has not been satisfactory $(1,6,32)$. Therefore, the management of aerodigestive fistulas remains challenging. The prognosis of malignant aerodigestive fistulas caused by esophageal cancer or lung cancer is poor $(5,11)$. For such patients, airway stenting is a palliative option that could alleviate clinical symptoms and improve quality of life by sealing the fistula $(14,33)$. Patients with benign aerodigestive fistulas after esophagectomy have longer expected survival, and thus, the aim of their treatment is complete healing of the fistula (11).

In the current study, the novel segmented covered metallic stent was used for malignant aerodigestive fistulas caused by lung cancer or esophageal cancer and benign aerodigestive fistulas after esophagectomy. Due to the involvement of the carina or distal bronchus, a Y-shaped stent was used. A tubular covered airway stent was not used considering the higher risk of stent migration and obstruction of the opening of the lobar bronchus. In this study, 9 patients with aerodigestive fistulas after esophagectomy achieved complete healing. In this study, the healing rate of fistulas was $34.6 \%$, which was close to that found in a previous study that reported the application of airway stents in thoracogastricairway fistula (34.5\%) (11). Healing of such fistulas is associated with several factors, such as the stent indwelling time, intervention time, and radiotherapy or chemotherapy $(1,11,30)$. For benign aerodigestive fistulas, insufficient indwelling time of the stent causes incomplete healing of the fistula, while a long stent indwelling time could lead to stentrelated complications $(30,34)$. Therefore, timely removal of stents should be performed when the fistula is identified to be healed. The interval between the diagnosis of benign aerodigestive fistulas and airway stenting of patients with a cured fistula was shorter than that of patients with an unhealed fistula, indicating the need for timely intervention, which was consistent with previous studies (11). None of the 10 malignant aerodigestive fistulas achieved healing in this study due to the invasion of a malignant tumor, which was also consistent with previous studies $(5,11)$. The sealing of the fistula by airway stenting can prevent food or water from entering the airway even if the fistula cannot be healed. In this study, fistulas in $80.77 \%$ of patients were sealed completely, slightly higher than previously reported (11).

According to previous studies, the carina angle of a normal person is $60-80^{\circ}$, with variations among different patients $(25,26,35)$. Fortunately, with the aid of 3D printing, the angle of the 2 branches of the Y-shaped stent was in accordance with the carina angle of each patient when manufacturing the stents. However, the carina angle is not static, and conversely, it changes $10^{\circ}$ or more during respiratory movement and in patients with malignant tumors around the carina $(25,26)$. Consequently, pressure may occur between the stent and the bronchial wall, leading to patient discomfort and stent-related complications. Therefore, the stents need to be as flexible as possible to fit the airway wall and reduce irritation. To address this issue, we designed a novel segmented stent. The connection of the three independent parts-the main stem and the two branches_of the stent was made of PTFE which is soft and has excellent chemical stability. However, quantitative characterization of the flexibility of the Y-shaped airway stent has not been reported previously. In this study, we measured the bending force and spring-back force of the branches of the stents. To our knowledge, this is the first in vitro study that focused on the flexibility of different Y-shaped airway stents and thus provided an insight into interactions between the stents and the bronchial wall when external forces pressed the stents. Compared with a wholly 

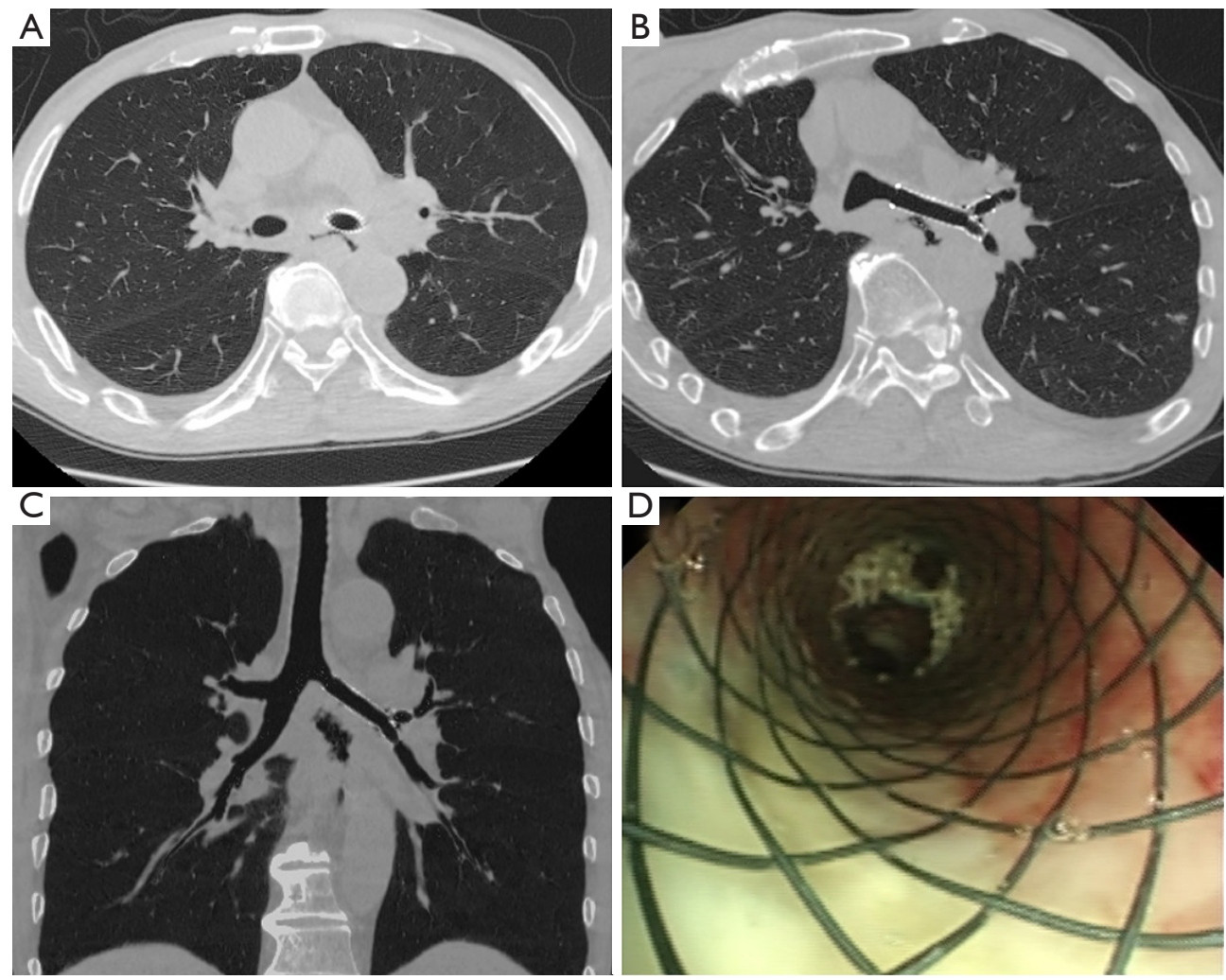

Figure 4 This is the same patient as presented in Figure 1. Follow-up CT and bronchoscopy 1 week after implantation of the stent. CT images (A,B,C) and bronchoscopy (D) showed that the fistula was completely sealed and the stent was patent. CT, computed tomography.

knitted stent, the connective part between the main stem and branches of the segmented stent has a smaller obstinacy modulus, making the stent more flexible and compliable with the movement of the airway. Therefore, the stent fitted to the airway wall more softly and tightly, and the pressure between the stent and the bronchial wall was lower, thereby the irritation to the airway wall was reduced, which may improve patient comfort and reduce the risk of granulation tissue proliferation.

The anatomical characteristics of the airway were different among patients. Anatomical variations of the bronchi may lead to mismatching of the stent with the airway, especially in the carina region and distal bronchus. Therefore, it is necessary to customize personalized stents to fit the anatomy of an individual patient's airway $(7,12,13)$. $3 \mathrm{D}$ printing technology has been used in various aspects of the medical field and has promoted the development of precision medicine. Previous studies have reported the application of $3 \mathrm{D}$ printing in designing personalized silicone airway stents for airway stenosis and tracheobronchomalacia $(15,16,36)$. However, few studies have reported the use of metallic stents customized with the assistance of $3 \mathrm{D}$ printing technology (9). Our study focused on aerodigestive fistulas involving the carina and distal bronchus, where the complex anatomy increased the difficulty of stenting. With the assistance of a 3D printed mold of the airway, the stent matched well with the individual geometry of the airway. The time required to manufacture stents needs to be shortened to fit the airway better in the future.

Timely recognition and treatment of complications are vital to achieving a successful clinical outcome. According to previous studies, the incidence of stent complications varied greatly depending on the type of airway stents $(27,37,38)$. In this study, the rates of sputum retention and granulation tissue proliferation were similar to those in previous studies that reported the application of covered metallic stents $(39,40)$. Bronchoscopy treatment and stent removal were effective in the treatment of granulation tissue proliferation. The dysfunction of cilia due to insertion of the covered stent caused sputum retention, which required aspiration under bronchoscopy. In this study, 1 patient had stent migration, which may be associated with various 

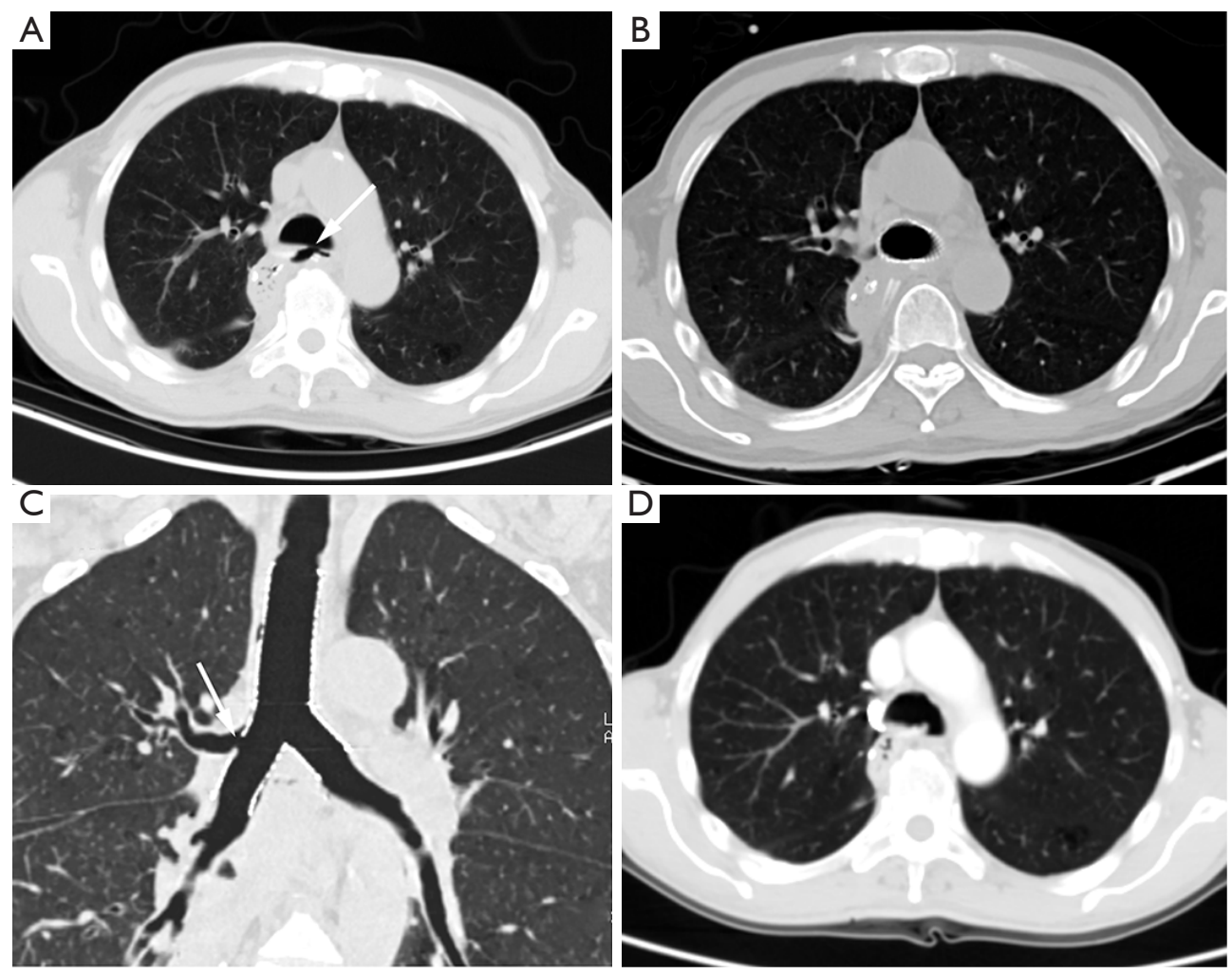

Figure 5 A 58-year-old man with thoracogastric-tracheal fistula after esophagectomy due to esophageal cancer. (A) Axial CT image before the stenting procedure showed that the trachea communicated with the thoracic stomach (white arrow); (B) axial CT image 1 month after the stenting procedure showed that the fistula was completely sealed; (C) coronal CT showed that the fenestration of the right branch of the stent matched with the opening of the right upper lobe bronchus (white arrow); (D) axial CT image 1 week after stent removal showed that the fistula was healed. CT, computed tomography.
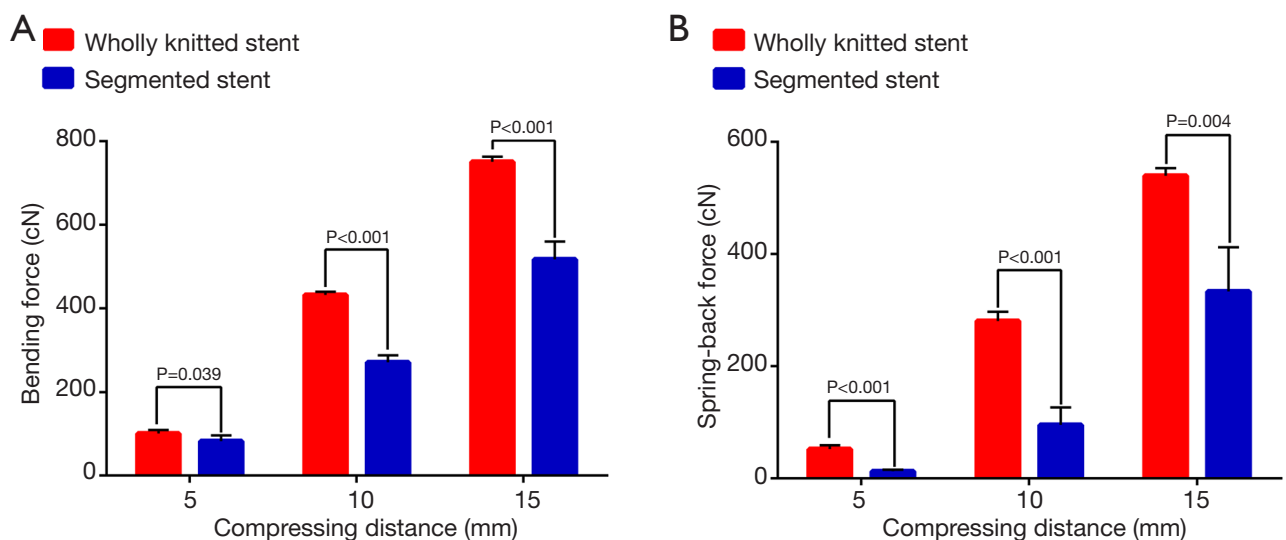

Figure 6 The bending force (A) and spring-back force (B) of the segmented stent were significantly lower than those of the wholly knitted stent when the compressing distances were 5,10 , and $15 \mathrm{~mm}$. 
factors, such as tumor progression and a rough cough. The Y-shaped stent is not prone to migration compared with the tubular stent. There is no current study on the migration rate of Y-shaped metallic stents with large sample size. The migration rate of this study was slighter lower than that of previous studies, which included several kinds of stents (5-20\%) (37,38). According to the in vitro test of flexibility, the segmented stent may comply with the movement of the bronchus better than a wholly knitted stent. Furthermore, whether the novel segmented stent might decrease the risk of migration and granulation tissue proliferation needs to be verified in further controlled studies. Due to potential complications, bronchoscopy and chest CT are warranted after airway stenting during follow-up to determine whether further management is required.

There are some limitations to our study. First, there was no control group in this study. Second, the sample size was small. A prospective controlled study with larger sample size is needed to compare segmented stents and wholly knitted stents.

In conclusion, this retrospective study demonstrated that a covered metallic segmented Y-shaped stent manufactured with the aid of $3 \mathrm{D}$ printing was feasible for treating complicated aerodigestive fistulas involving the carina and distal bronchus. The flexibility of the segmented metallic Y-shaped airway stent was better than that of the wholly knitted stent in an ex vivo setting.

\section{Acknowledgments}

Funding: This work was supported by the Shanghai key specialty construction project (No. ZK2019A02) and Clinical key specialist construction project of Shanghai municipal health commission [Interventional Radiology (No. shslczdzk06002) \& 3D Printing (No. shslczdzk07002)]. The sponsors of the study had no role in the study design, collection, analysis, interpretation of the data, writing the report, or deciding whether and where to submit the report for publication.

\section{Footnote}

Reporting Checklist: The authors have completed the STROBE (Strengthening the Reporting of Observational studies in Epidemiology) reporting checklist. Available at https://dx.doi.org/10.21037/atm-21-733

Data Sharing Statement: Available at https://dx.doi. org/10.21037/atm-21-733

Peer Review File: Available at https://dx.doi.org/10.21037/ atm-21-733

Conflicts of Interest: All authors have completed the ICMJE uniform disclosure form (available at https:// dx.doi.org/10.21037/atm-21-733). ZW reports grants from Shanghai key specialty construction project (No. ZK2019A02), and grants from Clinical key specialist construction project of Shanghai municipal health commission [Interventional Radiology (No. shslczdzk06002) \& 3D Printing (No. shslczdzk07002)]. In addition, ZW has a patent for a segmented bifurcated stent licensed. The other authors have no conflicts of interest to declare.

Ethical Statement: The authors are accountable for all aspects of the work in ensuring that questions related to the accuracy or integrity of any part of the work are appropriately investigated and resolved. The study was conducted in accordance with the Declaration of Helsinki (as revised in 2013). The study was approved by the Ethical Committee of the Ruijin Hospital/Luwan Branch (No. LWEC2019018) and individual consent for this retrospective analysis was waived.

Open Access Statement: This is an Open Access article distributed in accordance with the Creative Commons Attribution-NonCommercial-NoDerivs 4.0 International License (CC BY-NC-ND 4.0), which permits the noncommercial replication and distribution of the article with the strict proviso that no changes or edits are made and the original work is properly cited (including links to both the formal publication through the relevant DOI and the license). See: https://creativecommons.org/licenses/by-nc-nd/4.0/.

\section{References}

1. Zhou C, Hu Y, Xiao Y, et al. Current treatment of tracheoesophageal fistula. Ther Adv Respir Dis 2017;11:173-80.

2. Rodriguez AN, Diaz-Jimenez JP. Malignant respiratorydigestive fistulas. Curr Opin Pulm Med 2010;16:329-33.

3. Shin JH, Song HY, Ko GY, et al. Esophagorespiratory fistula: long-term results of palliative treatment with covered expandable metallic stents in 61 patients. Radiology 2004;232:252-9.

4. Buskens CJ, Hulscher JBF, Fockens P, et al. Benign 
tracheo-neo-esophageal fistulas after subtotal esophagectomy. Ann Thorac Surg 2001;72:221-4.

5. Shamji FM, Inculet R. Management of Malignant Tracheoesophageal Fistula. Thorac Surg Clin 2018;28:393-402.

6. Balakrishnan A, Tapias L, Wright CD, et al. Surgical Management of Post-Esophagectomy Tracheo-BronchialEsophageal Fistula. Ann Thorac Surg 2018;106:1640-6.

7. Han X, Li L, Zhao Y, et al. Individualized airway-covered stent implantation therapy for thoracogastric airway fistula after esophagectomy. Surg Endosc 2017;31:1713-8.

8. Li TF, Duan XH, Han XW, et al. Application of combined-type Y-shaped covered metallic stents for the treatment of gastrotracheal fistulas and gastrobronchial fistulas. J Thorac Cardiovasc Surg 2016;152:557-63.

9. Huang $W$, Shan $Q, W u Z$, et al. Retrievable covered metallic segmented $\mathrm{Y}$ airway stent for gastrorespiratory fistula of carina or main bronchi. J Thorac Cardiovasc Surg 2021;161:1664-1671.e2.

10. Herth FJF, Peter S, Baty F, et al. Combined airway and oesophageal stenting in malignant airway-oesophageal fistulas: a prospective study. Eur Respir J 2010;36:1370-4.

11. Wang H, Tao M, Zhang N, et al. Single application of airway stents in thoracogastric-airway fistula: results and prognostic factors for its healing. Ther Adv Respir Dis 2019;13:1753466619871523.

12. Sabath BF, Ost DE. Update on airway stents. Curr Opin Pulm Med 2018;24:343-9.

13. Freitag L, Gördes M, Zarogoulidis P, et al. Towards Individualized Tracheobronchial Stents: Technical, Practical and Legal Considerations. Respiration 2017;94:442-56.

14. Shan Q, Huang W, Shang M, et al. Customization of stent design for treating malignant airway stenosis with the aid of three-dimensional printing. Quant Imaging Med Surg 2021;11:1437-46.

15. Guibert N, Didier A, Moreno B, et al. Treatment of complex airway stenoses using patient-specific 3D-engineered stents: a proof-of-concept study. Thorax 2019;74:810-3.

16. Schweiger T, Gildea TR, Prosch H, et al. Patientspecific, 3-dimensionally engineered silicone Y-stents in tracheobronchomalacia: Clinical experience with a novel type of airway stent. J Thorac Cardiovasc Surg 2018;156:2019-21.

17. Guibert N, Didier A, Moreno B, et al. Treatment of Post-transplant Complex Airway Stenosis with a ThreeDimensional, Computer-assisted Customized Airway
Stent. Am J Respir Crit Care Med 2017;195:e31-3.

18. Dyet JF, Watts WG, Ettles DF, et al. Mechanical properties of metallic stents: how do these properties influence the choice of stent for specific lesions? Cardiovasc Intervent Radiol 2000;23:47-54.

19. Freitas AFDP, de Araujo MD, Zu WW, et al. Development of weft-knitted and braided polypropylene stents for arterial implant. J TEXT I 2010;101:1027-34.

20. Gyöngyösi M, Yang P, Khorsand A, et al. Longitudinal straightening effect of stents is an additional predictor for major adverse cardiac events. Austrian Wiktor Stent Study Group and European Paragon Stent Investigators. J Am Coll Cardiol 2000;35:1580-9.

21. Dong Z, Fu W, Wang Y, et al. Stent graft-induced new entry after endovascular repair for Stanford type B aortic dissection. J Vasc Surg 2010;52:1450-7.

22. Zou Q, Xue W, Lin J, et al. Mechanical characteristics of novel polyester/NiTi wires braided composite stent for the medical application. Results Phys 2016;6:440-6.

23. Hirdes MM, Vleggaar FP, de Beule $M$, et al. In vitro evaluation of the radial and axial force of self-expanding esophageal stents. Endoscopy 2013;45:997-1005.

24. Isayama H, Nakai $Y$, Toyokawa $Y$, et al. Measurement of radial and axial forces of biliary self-expandable metallic stents. Gastrointest Endosc 2009;70:37-44.

25. Chen JT, Putman CE, Hedlund LW, et al. Widening of the subcarinal angle by pericardial effusion. AJR Am J Roentgenol 1982;139:883-7.

26. Freitag L, Eicker R, Linz B, et al. Theoretical and experimental basis for the development of a dynamic airway stent. Eur Respir J 1994;7:2038-45.

27. Avasarala SK, Freitag L, Mehta AC. Metallic Endobronchial Stents. Chest 2019;155:1246-59.

28. Folch E, Keyes C. Airway stents. Ann Cardiothorac Surg 2018;7:273-83.

29. Park JH, Kim PH, Shin JH, et al. Removal of Retrievable Self-Expandable Metallic Tracheobronchial Stents: An 18Year Experience in a Single Center. Cardiovasc Intervent Radiol 2016;39:1611-9.

30. Kim JH, Shin JH, Song H, et al. Esophagorespiratory Fistula Without Stricture: Palliative Treatment with a Barbed Covered Metallic Stent in the Central Airway. J Vasc Interv Radiol 2011;22:84-8.

31. Kim YH, Shin JH, Song H, et al. Tracheal stricture and fistula: management with a barbed silicone-covered retrievable expandable nitinol stent. AJR Am J Roentgenol 2010;194:W232-7.

32. Reed MF, Mathisen DJ. Tracheoesophageal fistula.Chest 
Page 12 of 12

Surg Clin N Am 2003;13:271-89.

33. Shin JH, Kim J, Song H. Interventional Management of Esophagorespiratory Fistula. Korean J Radiol 2010;11:133-40.

34. Bi Y, Chen H, Li J, et al. Fluoroscopy-guided removal of individualised airway-covered stents for airway fistulas. Clin Radiol 2018;73:832.e1-832.e8.

35. Karabulut N. CT assessment of tracheal carinal angle and its determinants. Br J Radiol 2005;78:787-90.

36. Gildea TR, Young BP, Machuzak MS. Application of 3D Printing for Patient-Specific Silicone Stents: 1-Year Follow-Up on 2 Patients. Respiration 2018;96:488-94.

37. Herth FJF, Eberhardt R. Airway stent: what is new and what should be discarded. Curr Opin Pulm Med

Cite this article as: Shan Q, Huang W, Shang $M$, Wang Z, Xia N, Xue Q, Mao A, Ding X, Wang Z. Treatment of aerodigestive fistulas with a novel covered metallic Y-shaped segmented airway stent customized with the assistance of 3D printing. Ann Transl Med 2021;9(13):1051. doi: 10.21037/atm21-733
Shan et al. Treatment of aerodigestive fistula with airway stent

2016;22:252-6.

38. Casal RF. Update in airway stents. Curr Opin Pulm Med 2010;16:321-8.

39. Fortin M, Lacasse Y, Elharrar X, et al. Safety and Efficacy of a Fully Covered Self-Expandable Metallic Stent in Benign Airway Stenosis. Respiration 2017;93:430-5.

40. Kim MJ, Shin JH, Park JH, et al. Covered airway stent placement for malignant tracheobronchial strictures in patients with an endotracheal tube. Clin Radiol 2016;71:1120-5.

(English Language Editors: C. Betlazar-Maseh and J. Chapnick) 